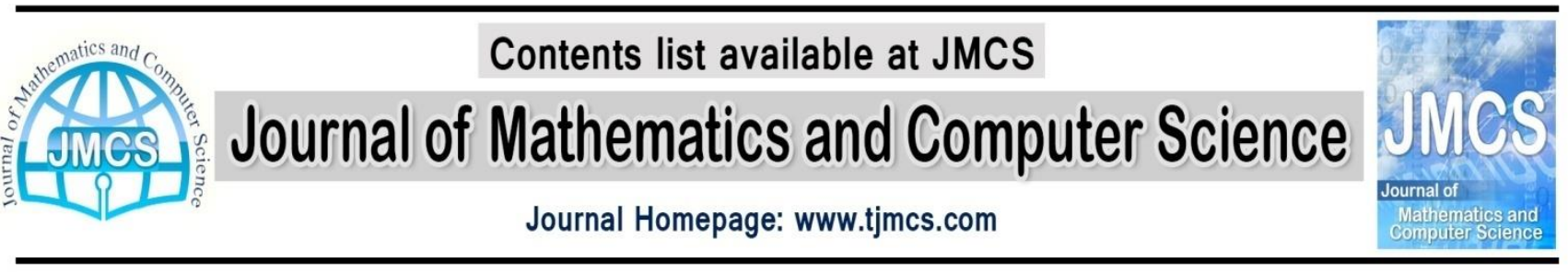

\title{
Fixed Point Theorems for Multi-valued Weakly C -contractive Mappings in Quasi-ordered Metric Spaces
}

\author{
E. Nazari \\ Department of Mathematics, Tafresh University, Tehran Ave. Tafresh, Iran. \\ nazari.esmaeil@gmail.com
}

Article history:

Received November 2014

Accepted December 2014

Available online January 2015

\section{Abstract}

The goal of this paper is to present some common fixed point theorems for multivalued weakly C-contractive mappings in quasi-ordered complete metric space. These results generalizes the existing fixed point results in the literature.

Keywords: Multivalued mapping, Hausdorff distance, Weakly C-contractive mapping, Common fixed point.

\section{Introduction}

Fixed point theory for contractive mapping first studied by Banach [1]. He proved that every contraction defined on a complete metric space has a unique fixed point. Since then the fixed point theory for single valued and multivalued mappings in metric space has been rapidly developed. In 1972, Chatterjea [2] introduce the concept of $C$-contraction as follows.

Definition1.1. A mapping $T: X \rightarrow X$ where $(\mathrm{X}, \mathrm{d})$ is a metric space is said to be a $C$ contraction if there exists $k \in(0,0.5)$ such that for all $x, y \in X$ the following inequality holds:

$$
d(T x, T y) \leq k((d(x, T y)+d(y, T x)) .
$$


Chatterjea [2] proved the following theorem:

Theorem1.1. Every C-contraction in a complete metric space has a unique fixed point.

Choudhury [3] introduce the concept of weakly $C$-contractive mapping as a generalization of $C$-contractive mapping and prove that every weakly $C$-contractive mapping in a complete metric space has a unique fixed point.

Definition1.2. Let $(\mathrm{X}, \mathrm{d})$ be a metric space. A mapping $T: X \rightarrow X$, is said to be weakly C-contractive if for all $x, y \in X$,

$$
d(T x, T y) \leq \frac{1}{2}(d(x, T y)+d(y, T x))-\varphi(d(x, T y), d(y, T x)),
$$

Where $\varphi:[0, \infty)^{2} \rightarrow[0, \infty)$ is a continuous function such that $\varphi(x, y)=0$ if and only $x=y=0$.

Harjani et al. [5] announced some fixed point results for weakly $C$-contractive mappings in a complete metric space endowed with a partial order. Meanwhile, Shatanawi [9] proved some fixed point theorems for a nonlinear weakly $C$-contraction type mapping in metric and ordered metric spaces. In this paper, we introduce the concept of multivalued weakly $C$-contractive mappings in quasi-ordered partial metric spaces and we prove some existence theorems of common fixed point for such mappings in the context of complete quasi-partial metric spaces under certain conditions.

\section{Preliminaries}

Let $(X, d, \leq)$ be a quasi-ordered metric space, with an order $\leq$ as a quasi-order (that is, a reflexive and transitive relation) and a metric d. Assume that $X$ having the following properties which appears in [8]:

(H1): if $\left\{x_{n}\right\}$ is a non-decreasing (resp. non-increasing) sequence in $X$ such that $x_{n} \rightarrow x$, then $x_{n} \leq x$ (resp. $\left.x_{n} \geq x\right)$ for all $n \in N$.

Let $2^{X}$ denote the family consisting of all nonempty subsets of $X$ we define the Hausdorff-Pseude metric in $H_{d}: 2^{X} \times 2^{X} \rightarrow \Re_{+} \cup\{\infty\}$ given by

$$
H_{d}(C, D)=\max \left\{\sup _{a \in C} d(a, D), \sup _{b \in D} d(C, b)\right\},
$$

where $d(a, D)=\inf _{b \in D} d(a, b), d(C, b)=\inf _{a \in C} d(a, b)$.

Definition2.1. Let $(X, d, \leq)$ be a quasi-ordered metric space. We say that $X$ is sequentially complete if every Cauchy sequence whose consecutive terms are comparable in $X$ converges.

Definition2.2. [6,7] Let $X$ be a quasi-ordered metric space. A subset $D \subseteq X$ is said to be approximative if the multivalued mapping 


$$
P_{D}(x)=\{y \in D: d(x, y)=d(D, x)\}, \quad \forall x \in X
$$

has nonempty values.

The multivalued mapping $T: X \rightarrow 2^{X}$ is said to have approximative values, $\mathrm{AV}$ for short, if $T x$ is approximative for each $x \in X$.

The multivalued mapping $T: X \rightarrow 2^{X}$ is said to have comparable approximative values, CAV for short, if $T$ has approximative values and, for each $z \in X$, there exists $y \in P_{T z}(x)$ such that $y$ is comparable to $z$.

The multivalued mapping $T: X \rightarrow 2^{X}$ is said to haveu pper comparable approximative values, UCAV, for short (resp: lower comparable approximative values, LCAV for short) if $T$ has approximative values and, for each $z \in X$, there exists $y \in P_{T z}(x)$ such that $y \geq z$ (resp: $y \leq z$ ). It is clear that $T$ has approximative values if it has compact values. In addition, if $T$ is single-valued, Then UCAV (LCAV) means that $T x \geq x(T x \leq x)$ for $x \in X$.

Definition2.3. The multivalued mappings $T, S$ are said to have a common fixed point if there is $x \in X$ such that $x \in T x$ and $x \in S x$.

In what follows, we give an analogy of the contraction which called multivalued $C$ weakly contraction mapping will play an important role in this sequel. To this end, we first introduce the following function.

Let $a \in(0, \infty], R_{a}^{+}=[0, a)$. let $f: \mathfrak{R}_{a}^{+} \rightarrow \mathfrak{R}$ satisfy,

(i) $f(0)=0$ and $f(t)>0$ for each $t \in(0, a)$

(ii) $f$ is non-decreasing on $\mathfrak{R}_{a}^{+}$

(iii) $f$ is continuous

(iv) $f(t+s) \leq f(t)+f(s)$ for $s, t \in \mathfrak{R}_{a}^{+}$.

For examples of such function $f$ we refer to (6).

Define

$$
\mathfrak{F}[0, a)=\{f \mid f \quad \text { satisfies (i)-(iv) above }\} .
$$

Let $a \in(0, \infty], \varphi: R_{a}^{+} \times R_{a}^{+} \rightarrow R^{+}$satisfy

(i) $\varphi(t, s)=0$ if and only if $s=t=0$.

(ii) $\varphi$ is continuous.

(iii) For any sequence $\left\{r_{n}\right\}$ with $\lim r_{n}=0$, there exist $a \in\left(0, \frac{1}{2}\right)$ and $n_{0} \in N$ such that $\varphi\left(r_{n}, 0\right) \geq(1-a) r_{n}\left(\right.$ or $\left.\varphi\left(0, r_{n}\right) \geq(1-\stackrel{n}{a}) r_{n}^{\infty}\right)$ for each $n \geq n_{0}$. Define 2 $\Phi([0, a) \times[0, a))=\{\varphi: \phi \quad$ satisfies(i)-(iii)above $\}$. 
Definition2.3. Let $X$ be a metric space and $d=\sup \{d(x, y): x, y \in X\}$. Set $a=d$ if $d=\infty$ and $a>d$ if $d<\infty$. Suppose the multivalued mappings $T, S: X \rightarrow 2^{X}, f \in \mathfrak{F}[0, a)$ and $\varphi \in \Phi([0, f(a-0)) \times[0, f(a-0)))$ satisfy

$$
f\left(H_{d}(T x, S y)\right) \leq f\left(\frac{1}{2}(d(x, S y)+d(y, T x))\right)-\varphi(f(d(x, S y)), f(d(y, T x)))
$$

For all $x, y \in X$ with $x$ and $y$ comparable. Then we say $T$ and $S$ satisfy weakly $C$ contraction with respect to $f$ and $\varphi$.

Definition2.4. For two subsets $A, B$ of $X$, we say that $A \leq_{r} B$ if, for each $a \in A$, there exists $b \in B$ such that $a \leq b$, and $A \leq B$ if each $a \in A$ and each $b \in B$ imply that $a \leq b$. A multi-valued mapping $T: X \rightarrow 2^{X}$ is said to be $r$-non-decreasing ( $r$-non-increasing) if $x \leq y$ implies that $T x \leq_{r} T y \quad\left(T y \leq_{r} T x\right)$ for all $x, y \in X . T$ is said to be $r$-monotone if $T$ is $r$-non-decreasing or $r$-non-increasing. The notion of non-decreasing (non-increasing) is similarly defined by writing $\leq$ instead of the notation $\leq_{r}$

\section{Main Result}

In this section we established common fixed point theorems for multivalued mappings on quasi-ordered complete metric spaces. The idea of the present theorem3.1 originate from the study of ananalogous problem for single-valued mappings in [4] and [9], and multivalued mappings in [6], [7] and [10].

Theorem3.1. Let $X$ be a quasi-ordered sequentially complete metric space and satisfy (H1). Suppose that the multivalued mappings $T$ and $S$ have UCAV and satisfy the weakly $C$-contraction with respect to $f$ and $\varphi$, then $T$ and $S$ have a common fixed point. Further, for each $x_{0} \in X$, the iterated sequence $\left\{x_{n}\right\}$ with $x_{2 n+1} \in T x_{2 n}$ and $x_{2 n+2} \in S x_{2 n+1}$ converges to the common fixed point of $T$ and $S$.

Proof: First we show that, if $T$ or $S$ has a fixed point it is a common fixed point of $T$ and $S$. Indeed, let $x$ be a fixed point of $T$ then we have,

$$
\begin{gathered}
f(d(x, S x)) \leq f\left(H_{d}(T x, S x)\right) \\
\leq f(0.5(d(x, S x)+d(x, T x)))-\varphi(f(d(x, S x)), f(d(x, T x))) \\
=f(0.5 d(x, S x))-\varphi(f(d(x, S x), 0) \\
\leq f(d(x, S x))-\varphi(f(d(x, S x), 0)
\end{gathered}
$$

This implies that, $\varphi(f(d(x, S x)), 0)=0$ and hence $f(d(x, S x))=0$ therefore $d(x, S x)=0$.

Since $x$ is $\mathrm{AV}$, therefore there exist $y \in P_{S x}(x)$ such that $d(y, x)=0$ i.e, $y=x$. Thus 
$x \in S x$. Let $x_{0} \in X$, if $x_{0} \in T x_{0}$ the proof is finished. Otherwise, from the fact that $T x_{0}$ has UCAV it follows there exists $x_{1} \in T x_{0}$ with $x_{1} \neq x_{0}$ and $x_{1} \geq x_{0}$ such that

$$
d\left(x_{0}, x_{1}\right)=\inf _{x \in x_{0}} d\left(x, x_{0}\right)=d\left(T x_{0}, x_{0}\right) .
$$

Again since $S x_{1}$ has UCAV it follows there exist $x_{2} \in S x_{1}$ with $x_{2} \neq x_{1}$ and $x_{2} \geq x_{1}$ such that

$$
d\left(x_{1}, x_{2}\right)=\inf _{x \in S x_{1}} d\left(x, x_{1}\right)=d\left(S x_{1}, x_{1}\right) .
$$

By induction and using UCAV, we can find in this way a sequence $\left\{x_{n}\right\}$ in $X$ with $x_{n+1} \geq x_{n}$ such that $x_{2 n+1} \in T x_{2 n}$ and

$$
d\left(x_{2 n+1}, x_{2 n}\right)=d\left(T x_{2 n}, x_{2 n}\right)
$$

and $x_{2 n+2} \in S x_{2 n+1}$ with

$$
d\left(x_{2 n+2}, x_{2 n+1}\right)=d\left(S x_{2 n+1}, x_{2 n+1}\right) .
$$

On the other hand

$$
\begin{gathered}
d\left(T x_{2 n}, x_{2 n}\right) \leq \sup _{x \in S x_{2 n-1}} d\left(T x_{2 n}, x\right) \\
\leq H_{d}\left(T x_{2 n}, S x_{2 n-1}\right) .
\end{gathered}
$$

Therefore

$$
d\left(x_{2 n+1}, x_{2 n}\right) \leq H_{d}\left(T x_{2 n}, S x_{2 n-1}\right) .
$$

Similarly we can show that

$$
d\left(x_{2 n+2}, x_{2 n+1}\right) \leq H_{d}\left(S x_{2 n+1}, T x_{2 n}\right) .
$$

Now we show that $\lim _{n \rightarrow \infty} d\left(x_{n+1}, x_{n}\right)=0$. By using (2) and since $f$ is non-decreasing, we have

$$
\begin{aligned}
& \quad f\left(d\left(x_{2 n+1}, x_{2 n+2}\right)\right) \leq f\left(H_{d}\left(T x_{2 n}, S x_{2 n+1}\right)\right) \\
& \leq f\left(0.5\left(d\left(x_{2 n}, S x_{2 n+1}\right)+d\left(x_{2 n+1}, T x_{2 n}\right)\right)^{2}\right)-\varphi\left(f\left(d\left(x_{2 n}, S x_{2 n+1}\right)\right), f\left(d\left(x_{2 n+1}, T x_{2 n}\right)\right)\right) \\
& \leq f\left(0.5\left(d\left(x_{2 n}, x_{2 n+2}\right)\right)-\varphi\left(f\left(d\left(x_{2 n}, x_{2 n+2}\right)\right), 0\right) \leq f\left(0.5 d\left(x_{2 n}, x_{2 n+2}\right)\right) .\right.
\end{aligned}
$$

As $f$ is a non-decreasing function, we get

$$
d\left(x_{2 n+1}, x_{2 n+2}\right) \leq 0.5 d\left(x_{2 n}, x_{2 n+2}\right) .
$$

Since 


$$
d\left(x_{2 n}, x_{2 n+2}\right) \leq d\left(x_{2 n}, x_{2 n+1}\right)+d\left(x_{2 n+1}, x_{2 n+2}\right) .
$$

We have

$$
d\left(x_{2 n+1}, x_{2 n+2}\right) \leq d\left(x_{2 n}, x_{2 n+1}\right) .
$$

Similarly, by using (1) one can show that

$$
d\left(x_{2 n}, x_{2 n+1}\right) \leq 0.5 d\left(x_{2 n-1}, x_{2 n+1}\right) .
$$

Thus

$$
d\left(x_{2 n}, x_{2 n+1}\right) \leq d\left(x_{2 n-1}, x_{2 n}\right) .
$$

From (4) and (6), we have

$$
d\left(x_{n}, x_{n+1}\right) \leq d\left(x_{n-1}, x_{n}\right), \quad \forall n \in N .
$$

So, by (7) we get that $\left\{d\left(x_{n}, x_{n+1}\right): n \in N\right\}$ is a non-increasing sequence. Hence there is $r \geq 0$ such that

$$
\lim _{n \rightarrow \infty} d\left(x_{n}, x_{n+1}\right)=r .
$$

By (3) and (5) we have

$$
\begin{aligned}
& d\left(x_{n}, x_{n+1}\right) \leq 0.5 d\left(x_{n-1}, x_{n+1}\right) \\
\leq & 0.5\left(d\left(x_{n-1}, x_{n}\right)+d\left(x_{n}, x_{n+1}\right)\right) .
\end{aligned}
$$

Letting $n \rightarrow \infty$ and using (8), we get that

$$
r \leq \lim _{n \rightarrow \infty} 0.5 d\left(x_{n-1}, x_{n+1}\right) \leq 0.5(r+r) .
$$

Hence

$$
\lim _{n \rightarrow \infty} d\left(x_{n-1}, x_{n+1}\right)=2 r .
$$

Using the continuity $f, \varphi$ and (3), we get that

$$
f(r) \leq f(0.5(2 r))-\varphi(f(2 r), 0)),
$$

which implies that $\varphi(f(2 r), 0)=0$ and hence $r=0$.

Next we show that $\left(x_{n}\right)$ is a Cauchy sequence in $X$. Since $\lim _{n \rightarrow \infty} f\left(d\left(x_{n-1}, x_{n+1}\right)\right)=0$, from assumption (iii) of $\varphi$ there exists $0<a<\frac{1}{2}$ and $n_{0} \in N$ such that

$$
\varphi\left(f\left(\left(d\left(x_{n-1}, x_{n+1}\right)\right), 0\right) \geq a f\left(d\left(x_{n-1}, x_{n+1}\right)\right) \quad \text { for all } n \geq n_{0}\right. \text {. }
$$


On the other hand, for any given $\epsilon>0$, we choose $\delta>0$ to be small enough such that $f(\delta)<\frac{a}{1-2 a} f(\epsilon)$. Moreover, there exists $n_{1}$ such that $d\left(x_{n+1}, x_{n}\right) \leq \delta$, for each $n \geq n_{1}$.

Now for any numbers $m>n \geq \max \left\{n_{0}, n_{1}\right\}$, from the inequality (1) and (2) we have

$$
\begin{gathered}
f\left(d\left(x_{n+1}, x_{n}\right)\right) \leq f\left(H_{d}\left(T x_{n}, S x_{n-1}\right)\right) \quad\left(\text { or } \quad f\left(H_{d}\left(T x_{n-1}, S x_{n}\right)\right)\right. \\
\leq f\left(0.5\left(d\left(x_{n}, S x_{n-1}\right)+d\left(x_{n-1}, T x_{n}\right)\right)\right) \\
-\varphi\left(f\left(d\left(x_{n}, S x_{n-1}\right)\right), f\left(d\left(x_{n-1}, T x_{n}\right)\right)\right) \\
\leq f\left(0.5\left(d\left(x_{n-1}, x_{n+1}\right)\right)\right)-\varphi\left(0, f\left(d\left(x_{n-1}, x_{n+1}\right)\right)\right) \\
\leq f\left(d\left(x_{n-1}, x_{n+1}\right)\right)-(1-a) f\left(d\left(x_{n-1}, x_{n+1}\right)\right) \\
\leq a f\left(d\left(x_{n-1}, x_{n+1}\right)\right) \\
\leq a\left(f\left(d\left(x_{n-1}, x_{n}\right)\right)+f\left(d\left(x_{n}, x_{n+1}\right)\right)\right) .
\end{gathered}
$$

Therefore

$$
f\left(d\left(x_{n}, x_{n+1}\right)\right) \leq(a /(1-a)) f\left(d\left(x_{n-1}, x_{n}\right)\right) .
$$

Set $\alpha=\frac{a}{1-a}<1$. By repeating this procedure, for any $k>n$ we obtain

$$
f\left(d\left(x_{k}, x_{k-1}\right)\right) \leq \alpha f\left(d\left(x_{k-1}, x_{k-2}\right)\right) \leq \ldots \leq a^{k-n} f\left(d\left(x_{n}, x_{n-1}\right)\right) .
$$

Therefore, from the assumption of $f$ we have,

$$
\begin{gathered}
f\left(d\left(x_{m}, x_{n}\right)\right) \leq f\left(d\left(x_{m}, x_{m-1}\right)\right)+f\left(d\left(x_{m-1}, x_{m-2}\right)\right)+\ldots+f\left(d\left(x_{n+1}, x_{n}\right)\right) \\
\leq \alpha^{m-n} f\left(d\left(x_{n}, x_{n-1}\right)\right)+\alpha^{m-n-1} f\left(d\left(x_{n}, x_{n-1}\right)\right)+\ldots \\
+\alpha f\left(\left(d\left(x_{n}, x_{n-1}\right)\right)\right. \\
=\left(\alpha-\alpha^{m-n+1} /(1-\alpha)\right) f\left(d\left(x_{n}, x_{n-1}\right)\right) \\
<(\alpha /(1-\alpha)) f\left(d\left(x_{n}, x_{n-1}\right)\right)<(\alpha /(1-\alpha)) f(\delta) \\
=(a /(1-2 a)) f(\delta)<f(\epsilon) .
\end{gathered}
$$

This shows that $d\left(x_{m}, x_{n}\right)<\epsilon$, so $\left\{x_{n}\right\}$ is a $\leq-$ non-decreasing Cauchy sequence. Since $X$ is a sequentially complete, there exists $x^{*} \in X$ such that $\lim _{n \rightarrow \infty} x_{n}=x^{*}$. Finally, we prove that $x^{*}$ is a common fixed point of $T$ and $S$. For every $n \in N,(H 1)$ guarantees that $x_{n}$ is comparable to $x^{*}$, so for $n \in N$ we have, 


$$
\begin{aligned}
& \quad f\left(d\left(x_{2 n+2}, S x^{*}\right)\right) \leq f\left(\sup _{x \in T_{2 n+1}} d\left(x, S x^{*}\right)\right) \leq f\left(H_{d}\left(T x_{2 n+1}, S x^{*}\right)\right) \\
& \leq f\left(0.5\left(d\left(x_{2 n+1}, S x^{*}\right)+d\left(x^{*}, T x_{2 n+1}\right)\right)\right)-\varphi\left(f\left(d\left(x_{2 n+1}, S x^{*}\right)\right), f\left(d\left(x^{*}, T x_{2 n+1}\right)\right)\right) \\
& \quad \leq f\left(0.5\left(d\left(x_{2 n+1}, S x^{*}\right)+d\left(x^{*}, x_{2 n+2}\right)\right)\right)-\varphi\left(f\left(d\left(x_{2 n+1}, S x^{*}\right)\right), f\left(d\left(x^{*}, x_{2 n+2}\right)\right)\right),
\end{aligned}
$$

Since $\varphi$ is 1.s.c, letting $n \rightarrow \infty$ in (9) we get

$$
f\left(d\left(x^{*}, S x^{*}\right)\right) \leq f\left(0.5 d\left(x^{*}, S x^{*}\right)\right)-\varphi\left(f\left(d\left(x^{*}, S x^{*}\right)\right), 0\right) .
$$

Which implies $\varphi\left(f\left(d\left(x^{*}, S x^{*}\right)\right), 0\right)=0$ and hence $d\left(x^{*}, S x^{*}\right)=0$. Since $S x^{*}$ is AV, there exist $y \in P_{S x^{*}}$. such that $d\left(y, x^{*}\right)=0$ i.e, $y=x^{*}$, therefore $x^{*} \in S x^{*}$, i.e $x^{*}$ is a fixed point of $S$, and so it is a common fixed point. This completes the proof.

Similar to the proof of Theorem 3.1 we have the following Theorem.

Theorem.3.2. Let $X$ be a sequentially complete quasi-ordered metric space and satisfy (H1). Suppose that $T, S: X \rightarrow 2^{X}$ be two mappings that satisfy weakly $C$-contraction with respect to $f$ and $\varphi$, and have LCAV. Then $T$ and $S$ have a common fixed point. Further, for each $x_{0} \in X$, the iterated sequence $\left\{x_{n}\right\}$ with $x_{2 n+1} \in T x_{2 n}$ and $x_{2 n+2} \in S x_{2 n+1}$ converges to the common fixed point of $T$ and $S$.

Theorem3.3. Let $X$ be an totally ordered sequentially complete metric space and satisfy (H1) and the following (H2) $x \leq y \leq z$ implies that $d(z, x) \geq d(y, x)$ for all $x, y, z \in X$.

Suppose that $T$ and $S$ satisfy all conditions given in Theorem 3.1 (resp. in Theorem 3.2), then $T, S$ have a unique common fixed point $x \in X$ and the iterated convergence of Theorem 3.1 holds.

Proof: Theorem 3.1 (resp. Theorem 3.2) ensures existence of common fixed points. To prove the uniqueness, let both $x$ and $y$ be common fixed point of $T$ and $S$. Since $(X, \leq)$ is a totally ordered space, we have either $x>y$ or $y>x$. Without loss of generality, we assume that the former is true. If T has UCAV, we have $x^{*} \in T x$, with $x \leq x^{*}$ and $d\left(x^{*}, y\right)=d(T x, y)$. From our assumption it follows that $d\left(x^{*}, y\right) \geq d(x, y)$. On the other 
hand, $x \in T x$ implies that $d\left(x^{*}, y\right) \leq d(x, y)$. Hence, $d\left(x^{*}, y\right)=d(x, y)=d(T x, y)$. If $x \neq y$, then $d(x, y)>0$. Thus

$$
d(x, y)=d(T x, y) \leq H_{d}(T x, S y)
$$

If $T$ has LCAV, so does $S$, we have $y^{*} \in S y$ with $y^{*} \leq y$ and $d\left(y^{*}, x\right)=d(S y, x)$. From (H2) it follows that $d\left(y^{*}, x\right) \geq d(x, y)$. On the other hand, $y \in S y$ implies that $d\left(y^{*}, x\right) \leq d(x, y)$. Hence, $d\left(y^{*}, x\right)=d(x, y)=d(x, S y)$. At all events, (10) holds if $x \neq y$.

$$
\begin{aligned}
f(d(x, y)) \leq f\left(H_{d}(T x, S y)\right) & \leq f\left(\frac{1}{2}(d(y, T x)+d(x, S y))\right)-\varphi(d(y, T x), d(x, S y)) \\
& =f(d(x, y))-\varphi(d(x, y), d(x, y))<f(d(x, y))
\end{aligned}
$$

This is a contradiction. Consequently, the inequality $x<y$ is not true. By the same methods we can verify that $y<x$ is also not true. Thus $x=y$.

Theorem.3.3. Let $X$ be a sequentially complete quasi-ordered metric space and satisfy (H1). Suppose that $T, S: X \rightarrow 2^{X}$ be two mappings have AV, are non-decreasing, and weak $C$-contraction with respect to $f$ and $\varphi$. If there exists $x_{0} \in X$ such that $\left\{x_{0}\right\} \leq S x_{0} \leq T x_{0}$. Then $T$ and $S$ have a common fixed point. Further, the iterated convergence of Theorem 3.1 holds.

Proof: let $x_{0} \in X$, if $x_{0} \in S x_{0}$ then is a common fixed point of $T$ and $S$ thus the proof is complete. Otherwise, since $S x$ has AV, there exist $x_{1} \in S x_{0}$ with $x_{1} \geq x_{0}$ and $d\left(x_{0}, x_{1}\right)=d\left(S x_{0}, x_{0}\right)$. Since $x \geq x_{1}$ for all $x \in T x_{1}$. If $x_{1} \in T x_{1}$, the proof is finished, otherwise, by means of $T x$ is $\mathrm{AV}$, there exist $x_{2} \in T x_{1}$ with $x_{2} \geq x_{1}$ and $d\left(x_{1}, x_{2}\right)=d\left(T x_{1}, x_{1}\right)$. Inductively, we can construct a sequence $x_{n}$ in $X$ as $x_{n} \neq x_{n-1}$ and $x_{n} \geq x_{n-1}$ such that $x_{2 n+1} \in T_{2 n}, x_{2 n+2} \in S x_{2 n+1}$ and (1), (2) hold. Now the rest of the proof is the same as theorem 3.1 .

\section{References}

[1] S. Banach, "Surles op'erations dans les ensembles abstraits et leurs application aux 'equationsint'egrales", Fund.Math. 3(1922), 133-181 (French).

[2] S. K. Chatterjea, "Fixed point theorems", C. R .Acad. Bulgare Sci. 25,727-730 (1972). 
[3] B. S. Choudhury, "Unique fixed point theorem for weakly C-contractive mappings", Kathmandu University Journal of Science, Engineering and Technology 5, 6-13 (2009).

[4] A. Choudhury, T. Som, "Few common fixed point results for weakly commuting mappings", Journal of Mathematics and Computer Science. 6 (2013), 27-35.

[5] J. Harjani, Lopez, B. Sadarangani, "Fixed point theorems for weakly C-contractive mappings in ordered metric spaces", Computers and Mathematics with Applications 61 (2011) 790-796.

[6] S. H. Hong, "Fixed points of multivalued operators in ordered metric spaces with applications", Nonlinear Anal. 72 (2010) 3929-3942.

[7] S. H. Hong, D. Guan, L. Wang, "Hybrid fixed points of multivalued operators in metric spaces with applications", Nonlinear Anal. 70 (2009) 4106-4117.

[8] J. J. Nieto, R. Rodrguez-Lopez, "Contractive mapping theorems in partially ordered sets and applications to ordinary differential equations", Order. 22 (2005), 223- 239.

[9] W. Shatanawi, "Fixed point theorems for nonlinear weakly C-contractive mappings in metric spaces", Mathematical and Computer Modelling. 54 (2011) 2816-2826.

[10] Z. Qiu, "Existence and uniqueness of common fixed points for two multivalued operators in ordered metric spaces", Computers and Mathematics with Applications 63, (2012), 1279-1286. 